\title{
OPERATOR RADII OF COMMUTING PRODUCTS
}

\author{
K. OKUBO AND T. ANDO
}

\begin{abstract}
ABSTRACr. Operator radii $w_{\rho}(T)$ for a bounded linear operator $T$ on a Hilbert space were introduced in connection with unitary $\rho$-dilations. We shall be concerned with universal estimates for the ratios
\end{abstract}

$$
w_{\rho}(S T) /\left(w_{\sigma}(S) \cdot w_{\rho}(T)\right)
$$

for commuting operators $S, T$ and $\sigma, \rho>0$.

1. All operators in this paper are bounded linear operators on a complex Hilbert space $\mathfrak{Q}$. We say an operator $T$ belongs to the class $e_{\rho}(0<\rho<\infty)$ if there exists a unitary operator $U$ on some Hilbert space $\Re$ such that $\mathscr{\Omega}$ contains $\mathfrak{Q}$ as a subspace and such that

$$
T^{n} h=\rho P U^{n} h \quad \text { for } h \in \mathfrak{Q} \text { and } n=1,2, \ldots,
$$

where $P$ is the orthogonal projection of $\mathscr{R}$ onto $\mathfrak{Q}$. The classes $\bigodot_{\rho}$ were defined by Sz.-Nagy and Foias [7] while Holbrook [4] introduced the operator radii $w_{\rho}(T)$ of an operator $T$, relative to $\bigodot_{\rho}$, by the formula:

$$
w_{\rho}(T)=\inf \left\{\gamma ; \gamma>0, \gamma^{-1} T \in \mathcal{C}_{\rho}\right\} \text {. }
$$

The family of operator radii includes the familiar quantities in operator theory: $w_{1}(T)=\|T\|($ norm of $T), w_{2}(T)=w(T):=\sup \{|(T h, h)| ;\|h\|=1\}$ (numerical radius of $T$ ), and $\lim _{\rho \rightarrow \infty} w_{\rho}(T)=r(T)$ (spectral radius of $T$ ).

For each $\rho>0$ the operator radius $w_{\rho}(\cdot)$ is a pseudonorm on $\mathfrak{B}(\mathfrak{Q})$, the space of all operators, in the sense that

$$
w_{\rho}(\alpha T)=|\alpha| w_{\rho}(T), \quad w_{\rho}(T+S) \leqslant \gamma_{\rho}\left\{w_{\rho}(T)+w_{\rho}(S)\right\}
$$

where $\gamma_{\rho}$ is a positive constant depending only on $\rho$. The constant $\gamma_{\rho}$ can be equal to 1 or $\rho$ according as $0<\rho \leqslant 2$ or $2<\rho<\infty$. Each operator $S$ induces a linear map $\curvearrowright$ on $\mathfrak{B}(\mathfrak{S})$ by the relation: ${ }_{\curvearrowright}(T)=S T$. When the space $\mathfrak{B}(\mathfrak{S})$ is provided with the operator radius $w_{\rho}(\cdot)$, then for each operator $S$ and each $\sigma>0$ the Lipschitz constant of the map 2 with respect to this pseudonorm $w_{\rho}(\cdot)$ is majorated by $\sigma(2-\rho) \cdot w_{\sigma}(S)$ or $\sigma \rho \cdot w_{\sigma}(S)$ according as $0<\rho \leqslant 1$ or $1<\rho<\infty$ (see the next section):

$$
w_{\rho}(S T) \leqslant \begin{cases}\sigma(2-\rho) \cdot w_{\sigma}(S) \cdot w_{\rho}(T) & \text { for } 0<\rho \leqslant 1, \\ \sigma \rho \cdot w_{\sigma}(S) \cdot w_{\rho}(T) & \text { for } 1<\rho<\infty .\end{cases}
$$

Received by the editors August 12, 1974 and, in revised form, March 3, 1975.

AMS (MOS) subject classifications (1970). Primary 47A10, 47A20, 47A30.

Key words and phrases. Operator radius, numerical radius, contraction, unitary $\rho$-dilation, class $\bigcup_{\rho}$, commuting operators, Hilbert space. 
We can expect, however, to have better estimates for these if the map 2 is confined to the commutant of $S$, the subspace of all operators commuting with $S$. Indeed, it has been conjectured (cf. [4], [5]) that if $S$ commutes with $T$ then

$$
w_{\rho}(S T) \leqslant \sigma \cdot w_{\sigma}(S) \cdot w_{\rho}(T)
$$

and, in particular,

$$
w_{\rho}(S T) \leqslant\|S\| w_{\rho}(T) .
$$

Remark that the inequality (**) implies, in its turn, the inequality (*) because of the general relation: $\|S\| \leqslant \sigma \cdot w_{\sigma}(S)$ for $0<\sigma<\infty$.

Holbrook [4] and, independently, Sz.-Nagy [6] showed that if $S$ double commutes with $T$, i.e. $S T=T S$ and $S T^{*}=T^{*} S$ then (**) holds.

Our purpose in this paper is to take a step towards the above inequalities. We shall prove that if $S$ commutes with $T$ then (*) is valid for $\sigma \geqslant 2$, and for $0<\sigma<2$

$$
w_{\rho}(S T) \leqslant L_{\sigma} \cdot w_{\sigma}(S) \cdot w_{\rho}(T)
$$

where $L_{\sigma}$ is an increasing function of $\sigma$ such that $L_{1}=\sqrt{2}, \lim _{\sigma \rightarrow 0} L_{\sigma}=0$ and $\lim _{\sigma \rightarrow 2} L_{\sigma}=2$. then

As to the second inequality (**), we shall show that if $S$ commutes with $T$

$$
w_{\rho}(S T) \leqslant K_{\rho} \cdot\|S\| \cdot w_{\rho}(T)
$$

where $K_{\rho}$ is an explicitly given constant such that $0<K_{\rho} \leqslant \rho, K_{1}=1$ and $K_{\rho}$ increases to $\sqrt{2}$ as $\rho \rightarrow \infty$.

2. First we recall some properties of operator radii used in this paper :

(i) (Sz.-Nagy and Foiaş [7]). $w_{\rho}(T) \leqslant 1$ if and only if $r(T) \leqslant 1$ and

$$
\operatorname{Re}_{e}\left[\rho+2 z T(1-z T)^{-1}\right] \geqslant 0 \text { for }|z|<1 \text {. }
$$

(ii) (Holbrook [4]). $w_{\rho}(\xi T)=|\xi| \cdot w_{\rho}(T)$ for all complex $\xi, w_{\rho}(T)=w_{\rho}\left(T^{*}\right)$, and $w_{\rho}(T)$ is a continuous nonincreasing function of $\rho$.

(iii) (Ando and Nishio [1]). $\rho \cdot w_{\rho}(T)=(2-\rho) \cdot w_{2-\rho}(T)$ for $0<\rho<2$, and $\rho \cdot w_{\rho}(T)$ is nondecreasing for $1 \leqslant \rho<\infty$.

Since $\|S\| \leqslant \sigma \cdot w_{\sigma}(S),\|T\| \leqslant \rho \cdot w_{\rho}(T)$ by (ii) and (iii) and $\|S T\|$ $\geqslant w_{\rho}(S T)$ for $1 \leqslant \rho<\infty$ by (ii), we have the following trivial estimate:

$$
w_{\rho}(S T) \leqslant\|S T\| \leqslant\|S\| \cdot\|T\| \leqslant \sigma \rho \cdot w_{\rho}(S) \cdot w_{\rho}(T) \text { for } 1<\rho<\infty .
$$

If $0<\rho \leqslant 1$ we can apply (iii) to get

$$
w_{\rho}(S T) \leqslant \sigma(2-\rho) \cdot w_{\sigma}(S) \cdot w_{\rho}(T) \text { for } 0<\rho \leqslant 1 .
$$

LEMMA 1. If $1 \leqslant \rho<\infty$, then $w_{\rho}(T) \leqslant 1$ implies

$$
\begin{aligned}
& \sum_{n=1}^{\infty} \alpha^{2 n}\left\|T^{n} h\right\|^{2} \\
& \quad \leqslant \frac{\rho \alpha^{2}\|h\|^{2}}{(1-\alpha)(2-\rho+\alpha \rho)} \quad \text { for } h \in \mathfrak{Q} \text { and } 1-2 / \rho<\alpha<1 .
\end{aligned}
$$


Proof. $1-z T$ has bounded inverse for $|z|<1$ and $\rho+2 z T(1-z T)^{-1}$ has positive real part by (i). It is well known that an operator $S$ has positive real part if and only if for each (and all) $\lambda>0$ the operator $S+\lambda$ has bounded inverse and $(S-\lambda)(S+\lambda)^{-1}$ has norm not greater than one. Applying this to $\rho+2 z T(1-z T)^{-1}$, we obtain

$$
\left\|\left\{\rho-\lambda+2 z T(1-z T)^{-1}\right\}\left\{\rho+\lambda+2 z T(1-z T)^{-1}\right\}^{-1}\right\| \leqslant 1,
$$

which implies

$$
\begin{array}{r}
\left\|\{(\rho-\lambda)-(\rho-\lambda-2) z T\}\{(\rho+\lambda)-(\rho+\lambda-2) z T\}^{-1} h\right\| \\
\leqslant\|h\| \quad \text { for } h \in \mathfrak{G} .
\end{array}
$$

Since

$$
\{(\rho+\lambda)-(\rho+\lambda-2) z T\}^{-1}=(\rho+\lambda)^{-1} \sum_{n=0}^{\infty}\left(\frac{\rho+\lambda-2}{\rho+\lambda} z T\right)^{n},
$$

we have, for $\lambda \neq \rho$,

$$
\begin{aligned}
& \{(\rho-\lambda)-(\rho-\lambda-2) z T\}\{(\rho+\lambda)-(\rho+\lambda-2) z T\}^{-1} \\
& \quad=\frac{\rho-\lambda}{\rho+\lambda}\left\{1+\frac{4 \lambda}{(\rho-\lambda)(\rho+\lambda-2)} \sum_{n=1}^{\infty}\left(\frac{\rho+\lambda-2}{\rho+\lambda} z T\right)^{n}\right\} .
\end{aligned}
$$

Therefore, setting $z=r e^{i \theta}$ and integrating the squares of both sides of (3) over $(0,2 \pi]$, we have

$$
\begin{gathered}
\frac{1}{2 \pi} \int_{0}^{2 \pi}\left\|h+\frac{4 \lambda}{(\rho-\lambda)(\rho+\lambda-2)} \sum_{n=1}^{\infty}\left(\frac{\rho+\lambda-2}{\rho+\lambda} r e^{i \theta} T\right)^{n} h\right\|^{2} d \theta \\
\leqslant\left(\frac{\rho+\lambda}{\rho-\lambda}\right)^{2}\|h\|^{2},
\end{gathered}
$$

and further, using the orthogonality relation of $e^{i n \theta}$ and $e^{i m \theta}(n \neq m)$ and letting $r \rightarrow 1$,

$$
\sum_{n=1}^{\infty}\left(\frac{\rho+\lambda-2}{\rho+\lambda}\right)^{2 n}\left\|T^{n} h\right\|^{2} \leqslant\left\{\left(\frac{\rho+\lambda}{\rho-\lambda}\right)^{2}-1\right\}\left\{\frac{(\rho-\lambda)(\rho+\lambda-2)}{4 \lambda}\right\}^{2}\|h\|^{2},
$$

hence

$$
\sum_{n=1}^{\infty}\left(\frac{\rho+\lambda-2}{\rho+\lambda}\right)^{2 n}\left\|T^{n} h\right\|^{2} \leqslant \frac{\rho(\rho+\lambda-2)^{2}}{4 \lambda}\|h\|^{2} .
$$

Since $\lambda$ can vary over $(0, \infty) \backslash\{\rho\}, \alpha \equiv(\rho+\lambda-2)(\rho+\lambda)^{-1}$ can move over $(1-2 / \rho, 1) \backslash\{1-1 / \rho\}$. We can conclude, replacing $(\rho+\lambda-2)(\rho+\lambda)^{-1}$ by $\alpha$ in (4), that (1) is valid except for $\alpha=1-1 / \rho$. This exception can, however, be removed by limit procedure.

Remark that the inequality (1) for the special case $\alpha=1-1 / \rho$ was obtained by Berger and Stampfli [2] by a different method.

Lemma 2. If $1<\rho<\infty$ and 


$$
\sum_{n=1}^{\infty} \rho^{-n}(\rho-1)^{n}\left|\left(T^{n} h, g\right)\right| \leqslant(\rho-1)\|h\| \cdot\|g\| \cdot \text { for } h, g \in \mathfrak{Q}
$$

then $w_{\rho}(T) \leqslant 1$.

Proof. First of all, (5) implies $\left\|T^{n}\right\|^{1 / n} \leqslant \rho(\rho-1)^{1 / n-1}$, hence $r(T)$ $\leqslant \rho(\rho-1)^{-1}$, for the spectral radius $r(T)$ is equal to $\lim _{n \rightarrow \infty}\left\|T^{n}\right\|^{1 / n}$. Then for each $|z|<1$ the series $\sum_{n=1}^{\infty}\left\{\rho^{-1}(\rho-1) z T\right\}^{n}$ is uniformly convergent to $\rho^{-1}(\rho-1) z T\left\{1-\rho^{-1}(\rho-1) z T\right\}^{-1}$, and by (5)

$$
\left|\left(z T\{\rho-(\rho-1) z T\}^{-1} h, g\right)\right| \leqslant\|h\| \cdot\|g\| \quad \text { for } h, g \in \mathfrak{Q},
$$

or equivalently

$$
\left\|z T\{\rho-(\rho-1) z T\}^{-1} h\right\| \leqslant\|h\| \quad \text { for } h \in \mathfrak{Q} \text { and }|z|<1,
$$

which is just (3) with $\lambda=\rho$. If $r(T) \leqslant 1$, then (6) can be converted to (2) with $\lambda=\rho$, hence $w_{\rho}(T) \leqslant 1$ by (i) as remarked in the proof of Lemma 1 . Now let us show that $(6)$ implies $r(T) \leqslant 1$. In fact, if $\xi$ is an approximate eigenvalue such that $|\xi|=r(T)>1$, take $|z|<1$ such that $z \xi=1+\varepsilon$ for some $\varepsilon>0$ small enough so that $\rho-(\rho-1)(1+\varepsilon)>0$. Then (6) implies

$$
1+\varepsilon=|z \xi| \leqslant|\rho-(\rho-1) z \xi|=\rho-(\rho-1)(1+\varepsilon)
$$

or $0 \leqslant-\rho \varepsilon$, a contradiction.

THEOREM 1. If $S$ commutes with $T$, i.e. $S T=T S$ then

$$
w_{\rho}(S T) \leqslant L_{\sigma} \cdot w_{\sigma}(S) \cdot w_{\rho}(T) \text { for } 0<\sigma, \rho<\infty
$$

where

$$
L_{\sigma}= \begin{cases}\frac{\sigma-1+\left(1+2 \sigma-\sigma^{2}\right)^{1 / 2}}{2-\sigma} & \text { for } 0<\sigma \leqslant 1, \\ \frac{1-\sigma+\left(1+2 \sigma-\sigma^{2}\right)^{1 / 2}}{2-\sigma} & \text { for } 1<\sigma<2, \\ \sigma & \text { for } 2 \leqslant \sigma<\infty .\end{cases}
$$

Proof. Since $\sigma \cdot w_{\sigma}(S)=(2-\sigma) \cdot w_{2-\sigma}(S)$ for $0<\sigma<1$ by (iii) and $w_{\sigma}(\lambda S)=\lambda \cdot w_{\sigma}(S)$ for $\lambda>0$ by (ii) and similarly for $\rho$ and $T$, we may assume that $1 \leqslant \sigma, \rho$ and $w_{\sigma}(S)=w_{\rho}(T)=1$. Given $\beta>1$, by Lemma 2 , a sufficient condition for $w_{\rho}(S T) \leqslant \beta$ is that

$$
\sum_{n=1}^{\infty} \beta^{-n} \rho^{-n}(\rho-1)^{n}\left|\left((S T)^{n} h, g\right)\right| \leqslant(\rho-1)\|h\| \cdot\|g\| \quad \text { for } h, g \in \mathfrak{B} \text {. }
$$

Since $S T=T S$ implies

$$
\left|\left((S T)^{n} h, g\right)\right|=\left|\left(T^{n} h, S^{* n} g\right)\right| \leqslant\left\|T^{n} h\right\| \cdot\left\|S^{* n} g\right\|,
$$

a sufficient condition for $w_{\rho}(S T) \leqslant \beta$ is

(7) $\sum_{n=1}^{\infty} \beta^{-n} \rho^{-n}(\rho-1)^{n}\left\|T^{n} h\right\| \cdot\left\|S^{* n} g\right\| \leqslant(\rho-1)\|h\| \cdot\|g\| \quad$ for $h, g \in \mathfrak{Q}$.

To get an estimate for the left side of (7), take $\gamma$ such that 
(8) $1-2 / \rho<1-\gamma / \rho<1$ and $1-2 / \sigma<(\rho-1) / \beta(\rho-\gamma)<1$.

Then we have by Lemma 1

$$
\sum_{n=1}^{\infty}\left(1-\frac{\gamma}{\rho}\right)^{2 n}\left\|T^{n} h\right\|^{2} \leqslant \frac{(\rho-\gamma)^{2}}{\gamma(2-\gamma)}\|h\|^{2}
$$

and by (ii): $w_{\sigma}\left(S^{*}\right)=w_{\sigma}(S)=1$,

$$
\begin{aligned}
\sum_{n=1}^{\infty}\left(\frac{\rho-1}{\beta(\rho-\gamma)}\right)^{2 n}\left\|S^{* n} g\right\|^{2} & \\
& \leqslant \frac{\sigma(\rho-1)^{2}\|g\|^{2}}{\{\beta(\rho-\gamma)-(\rho-1)\}\{(2-\sigma) \beta(\rho-\gamma)+\sigma(\rho-1)\}} .
\end{aligned}
$$

Applying the Schwartz inequality, we obtain from (9) and (10)

$$
\begin{aligned}
& \left\{\sum_{n=1}^{\infty} \beta^{-n} \rho^{-n}(\rho-1)^{n}\left\|T^{n} h\right\| \cdot\left\|S^{* n} g\right\|\right\}^{2} \\
& \quad \leqslant \sum_{n=1}^{\infty}\left(1-\frac{\gamma}{\rho}\right)^{2 n}\left\|T^{n} h\right\|^{2} \cdot \sum_{n=1}^{\infty}\left(\frac{\rho-1}{\beta(\rho-\gamma)}\right)^{2 n}\left\|S^{* n} g\right\|^{2} \\
& \quad \leqslant \frac{\sigma(\rho-\gamma)^{2}(\rho-1)^{2}\|h\|^{2} \cdot\|g\|^{2}}{\gamma(2-\gamma)\{\beta(\rho-\gamma)-(\rho-1)\}\{(2-\sigma) \beta(\rho-\gamma)+\sigma(\rho-1)\}} .
\end{aligned}
$$

Now a sufficient condition for $w_{\rho}(S T) \leqslant \beta$ is that the inequality

$$
\begin{aligned}
& (2-\sigma)(\rho-\gamma)^{2} \beta^{2}-2(\rho-1)(\rho-\gamma)(1-\sigma) \beta \\
& -\sigma(\rho-1)^{2}-\sigma \cdot \gamma^{-1}(2-\gamma)^{-1}(\rho-\gamma)^{2} \geqslant 0
\end{aligned}
$$

has a solution $\gamma$ satisfying (8), for then (7) holds.

Now if $1 \leqslant \sigma \leqslant 2$ and $\rho>1$, then (8) is satisfied with $\gamma=1$ and (11) takes the following form

$$
(2-\sigma) \beta^{2}-2(1-\sigma) \beta-2 \sigma \geqslant 0
$$

or equivalently

$$
\beta \geqslant \begin{cases}\frac{1-\sigma+\left(1+2 \sigma-\sigma^{2}\right)^{1 / 2}}{2-\sigma} & \text { for } 1 \leqslant \sigma<2, \\ 2 & \text { for } \sigma=2 .\end{cases}
$$

This proves the assertion for $0<\sigma \leqslant 2$. Finally the assertion for $\sigma>2$ results from the above by (iii), i.e. $\sigma \cdot w_{\sigma}(S) \geqslant 2 \cdot w_{2}(S)$ for $\sigma>2$.

It is quite easy, to show that $L_{\sigma}$ is a nondecreasing function of $\sigma$ on $[1, \infty)$ while $L_{\sigma} / \sigma$ decreases to 1 as $\sigma$ increases to 2 .

Holbrook [4, Theorem 6.3] proved that if $S$ commutes with $T$ then

$$
w_{\rho}(S T) \leqslant 2 w_{\rho}(S) \cdot w_{\rho}(T) \quad \text { for } 0<\rho \leqslant 2 .
$$

Theorem 1 gives an improvement because $L_{\sigma}<2$ for $0<\sigma<2$. On the other hand, under the assumption of double commutativity, i.e. $S T=T S$ and $S T^{*}=T^{*} S$, Holbrook [4, Theorem 6.2] proved the inequality: 


$$
w_{\rho}(S T) \leqslant \rho \cdot w_{\rho}(S) \cdot w_{\rho}(T) \text { for } 0<\rho<\infty .
$$

Our Theorem 1 shows that double commutativity can be replaced by mere commutativity for $2 \leqslant \rho<\infty$.

3. As remarked in the preceding section,

$$
w_{\rho}(S T) \leqslant \rho \cdot\|S\| \cdot w_{\rho}(T) \text { for } 1 \leqslant \rho<\infty .
$$

Holbrook [4], [5] pointed out that for each $\rho>1$ there is a constant $K_{\rho}<\rho$ such that if $S$ commutes with $T$

$$
w_{\rho}(S T) \leqslant K_{\rho} \cdot\|S\| \cdot w_{\rho}(T) .
$$

Further he showed that if we confine ourselves to commuting operators on a Hilbert space of fixed finite dimension then $K_{\rho}$ can be chosen so that $K_{\rho} / \rho \rightarrow 0$ as $\rho \rightarrow \infty$. But he could not get any explicit form of $K_{\rho}$. The following theorem gives an estimate for $K_{\rho}$ and, at the same time, removes the restriction on dimension.

THEOREM 2. If $S$ commutes with $T$, then

$$
w_{\rho}(S T) \leqslant K_{\rho} \cdot\|S\| \cdot w_{\rho}(T) \text { for } 0<\rho<\infty,
$$

where

$$
K_{\rho}= \begin{cases}\inf _{0<\gamma<1}\left\{\frac{1}{\gamma(2-\gamma)}+\frac{(\rho-1)^{2}}{(2-\rho-\gamma)^{2}}\right\}^{1 / 2} & \text { for } 0<\rho<1, \\ \inf _{0<\gamma<1}\left\{\frac{1}{\gamma(2-\gamma)}+\frac{(\rho-1)^{2}}{(\rho-\gamma)^{2}}\right\}^{1 / 2} & \text { for } 1 \leqslant \rho<\infty .\end{cases}
$$

Proof. As in the proof of Theorem 1, we may assume that $\|S\|=w_{\rho}(T)$ $=1$ and $\rho>1$. Now (8) with $\sigma=1$ is reduced to

$$
0<\gamma<\min \left\{2, \rho-\beta^{-1}(\rho-1)\right\},
$$

while (11) takes the form:

$$
(\rho-\gamma)^{2} \beta^{2}-(\rho-1)^{2}-\gamma^{-1}(2-\gamma)^{-1}(\rho-\gamma)^{2} \geqslant 0 .
$$

Since every $0<\gamma<1$ satisfies (12), we get the assertion from (13), just as in the proof of Theorem 1 .

Since, for each fixed $\gamma$ with $0<\gamma<1,(\rho-1)(\rho-\gamma)^{-1}$ is an increasing function of $1 \leqslant \rho<\infty$, the function $K_{\rho}$ is increasing and satisfies

$$
K_{1}=1, \quad K_{2}=\sqrt{27} / 4 \text { and } \lim _{\rho \rightarrow \infty} K_{\rho}=\sqrt{2}
$$

and

$$
\lim _{\rho \rightarrow \infty} K_{\rho} / \rho=0 .
$$

Obviously $K_{\rho} \leqslant \sqrt{2}<\rho$ for $2 \leqslant \rho<\infty$. Let $1<\rho<2$. Setting $\gamma=\rho-$ $(\rho-1)^{1 / 2}$ in $1 / \gamma(2-\gamma)+(\rho-1)^{2} /(\rho-\gamma)^{2}$, we see

$$
K_{\rho}^{2} \leqslant \rho-1+\left\{\rho-(\rho-1)^{1 / 2}\right\}^{-1}\left\{2-\rho+(\rho-1)^{1 / 2}\right\}^{-1}<\rho^{2} .
$$


4. When specialized to $\rho=2$, the most important and interesting case, Theorem 2 tells us that if $S$ commutes with $T$ then

$$
w(S T) \leqslant(\sqrt{27} / 4) \cdot\|S\| \cdot w(T) .
$$

Of course, $\sqrt{27} / 4 \simeq 1.2990$ is not at all the best possible estimate for $K_{2}$. In fact, M. J. Crabb informed us, in a private communication, of an effective method of getting better estimates for $K_{2}$. Let us sketch his idea.

Suppose that $S T=T S,\|S\|=w(T)=1$ and $h$ is a unit vector. Let

$$
\alpha_{n}=\left\|T^{n} h\right\|^{2}+\left\|T^{* n} h\right\|^{2} \quad(n=1,2, \ldots)
$$

and

$$
\beta_{n}=\alpha_{2^{n-1}} \quad(n=1,2, \ldots) .
$$

By the Schwartz inequality

$$
\begin{aligned}
4|\operatorname{Re}(S T h, h)|^{2} & =\left|\left(S T h+T^{*} S^{*} h, h\right)\right|^{2} \leqslant\left\|S T h+T^{*} S^{*} h\right\|^{2} \\
& =\|S T h\|^{2}+\left\|T^{*} S^{*} h\right\|^{2}+2 \operatorname{Re}\left((S T)^{2} h, h\right) .
\end{aligned}
$$

Since $S T=T S$ and $\|S\| \leqslant 1$, we have

$$
\begin{aligned}
4|\operatorname{Re}(S T h, h)|^{2} & \leqslant\|T h\|^{2}+\left\|T^{*} h\right\|^{2}+2\left|\operatorname{Re}\left(S^{2} T^{2} h, h\right)\right| \\
& \leqslant \beta_{1}+2\left|\operatorname{Re}\left(S^{2} T^{2} h, h\right)\right| .
\end{aligned}
$$

Applying the same method to $S^{2} T^{2}$ and so on, we have

$$
\begin{aligned}
& 2|\operatorname{Re}(S T h, h)| \\
& \leqslant\left\{\beta_{1}+\left\{\beta_{2}+\left\{\beta_{3}+\cdots\right.\right.\right. \\
& \left.\left.\left.\quad+\left\{\beta_{n}+2\left|\operatorname{Re}\left(S^{2 n} T^{2 n} h, h\right)\right|\right\}^{1 / 2}\right\}^{1 / 2}\right\}^{1 / 2} \cdots\right\}^{1 / 2} \\
& \leqslant\left\{\beta_{1}+\left\{\beta_{2}+\left\{\beta_{3}+\cdots+\left\{2 \beta_{n}\right\}^{1 / 2}\right\}^{1 / 2}\right\}^{1 / 2} \cdots\right\}^{1 / 2}
\end{aligned}
$$

because

$$
\begin{aligned}
2\left|\operatorname{Re}\left(S^{2^{n}} T^{2^{n}} h, h\right)\right| & \leqslant 2\left\|S^{2^{n-1}} T^{2^{n-1}} h\right\| \cdot\left\|S^{* 2^{n-1}} T^{* 2^{n-1}} h\right\| \\
& \leqslant\left\|T^{2^{n-1}} h\right\|^{2}+\left\|T^{* 2^{n-1}} h\right\|^{2}=\beta_{n} .
\end{aligned}
$$

Now $w(T) \leqslant 1$ implies

$$
\operatorname{Re}\left(e^{i \theta} T g, g\right) \leqslant(g, g) \text { for } g \in \mathfrak{Q} .
$$

Integrating this inequality over $0<\theta \leqslant 2 \pi$ with

$$
g=\xi_{2} e^{-2 i \theta} T^{* 2} h+\xi_{1} e^{-i \theta} T^{*} h+\xi_{0} h+\xi_{1} e^{i \theta} T h+\xi_{2} e^{2 i \theta} T^{2} h
$$

and using the orthogonality of $\left\{e^{i k \theta}\right\}$ we obtain

$$
\operatorname{Re}\left(\xi_{0} \bar{\xi}_{1} \alpha_{1}+\xi_{1} \bar{\xi}_{2} \alpha_{2}\right) \leqslant\left|\xi_{0}\right|^{2}+\left|\xi_{1}\right|^{2} \alpha_{1}+\left|\xi_{2}\right|^{2} \alpha_{2}
$$

for arbitrary complex numbers $\xi_{k}$. An elementary argument reveals that (14) 
simply asserts $\alpha_{2} \leqslant \alpha_{1}\left(4-\alpha_{1}\right)$. Hence, noting that $\beta_{1}=\alpha_{1}$ and $\beta_{2}=\alpha_{2}$, we are led to the inequalities:

$$
\begin{aligned}
|\operatorname{Re}(S T h, h)| & \leqslant \frac{1}{2}\left\{\alpha_{1}+\left\{2 \alpha_{2}\right\}^{1 / 2}\right\}^{1 / 2} \\
& \leqslant \frac{1}{2}\left\{\alpha_{1}+\sqrt{2}\left\{\alpha_{1}\left(4-\alpha_{1}\right)\right\}^{1 / 2}\right\}^{1 / 2} \\
& \leqslant \max _{0<x<4} \frac{1}{2}\left\{x+\sqrt{2}\{x(4-x)\}^{1 / 2}\right\}^{1 / 2} \\
& =\frac{1}{2}\{2+2 \sqrt{3}\}^{1 / 2} \simeq 1.169 .
\end{aligned}
$$

Since $e^{i \theta} S$, for any $\theta$, may replace $S$ in the above, we obtain $w(S T) \leqslant 1.169$. This method may be extended to obtain, in principle, a sequence of estimates for $w(S T)$. It is easy to generalize the argument leading to (14) to obtain

$$
\operatorname{Re}\left\{-\sum_{k=1}^{n} \xi_{k-1} \bar{\xi}_{k} \alpha_{k}\right\} \leqslant\left|\xi_{0}\right|^{2}+\sum_{k=1}^{n}\left|\xi_{k}\right|^{2} \alpha_{k}
$$

for an arbitrary complex sequence $\left\{\xi_{k}\right\}$. Then as in Crabb [3] we may conclude that

for some sequence

$$
\alpha_{k}=2\left\{\gamma_{k-1} \pm\left(\gamma_{k-1}^{2}-\gamma_{k-1} \gamma_{k}\right)^{1 / 2}\right\}
$$

Letting $\delta_{0}=1$ and $\delta_{k}=\gamma_{2^{k-1}}$, we have

$$
1=\gamma_{0} \geqslant \gamma_{1} \geqslant \cdots \geqslant 0 \text {. }
$$

$$
\beta_{k}=\alpha_{2^{k-1}} \leqslant 2\left\{\delta_{k-1}+\left(\delta_{k-1}^{2}-\delta_{k-1} \delta_{k}\right)^{1 / 2}\right\},
$$

so that, for each $n, w(S T)$ is bounded above by the maximum of

$$
\begin{aligned}
\frac{1}{2}\left\{2 \delta_{0}+2\left(\delta_{0}^{2}-\delta_{0} \delta_{1}\right)^{1 / 2}\right. & \\
& \left.+\left\{\cdots+\left\{4 \delta_{n-1}+4\left(\delta_{n-1}^{2}-\delta_{n-1} \delta_{n}\right)^{1 / 2}\right\}^{1 / 2} \cdots\right\}^{1 / 2}\right\}
\end{aligned}
$$

subject to the restriction:

$$
\begin{aligned}
& 1=\delta_{0} \geqslant \delta_{1} \geqslant \cdots \geqslant \delta_{n} \geqslant 0 \\
& \text { REFERENCES }
\end{aligned}
$$

1. T. Ando and K. Nishio, Convexity properties of operator radii associated with unitary p-dilations, Michigan Math. J. 20(1973), 303-307.

2. C. A. Berger and J. G. Stampf̣li, Norm relations and skew dilations, Acta Sci. Math. (Szeged) 28(1967), 191-195. MR 37 \#4661.

3. M. J. Crabb, The powers of an operator of numerical radius one, Michigan Math. J. 18(1971), 253-256. MR 44 \# 7345.

4. J. A. R. Holbrook, On the power-bounded operators of Sz.-Nagy and Foias, Acta Sci. Math. (Szeged) 29(1968), 299-310. MR 39 \#810.

5. gan Math. J. 18(1971), 149-159. MR 43 \#6755.

6. B. Sz.-Nagy, Products of operators of classes $C_{\rho}$, Rev. Roumaine Math. Pures Appl. 13(1968), 897-899. MR 39 \# 812.

7. B. Sz.-Nagy and C. Foias, On certain classes of power-bounded operators in Hilbert space, Acta Sci. Math. (Szeged) 27(1966), 17-25. MR 33 \#6413. 